PROCEEDINGS OF THE

AMERICAN MATHEMATICAL SOCIETY

Volume 128, Number 2, Pages 427-431

$\mathrm{S}$ 0002-9939(99)05383-6

Article electronically published on September 27, 1999

\title{
SOBOLEV SPACES, DIMENSION, AND RANDOM SERIES
}

\author{
ROBERT KAUFMAN \\ (Communicated by Frederick W. Gehring)
}

\begin{abstract}
We investigate dimension-increasing properties of maps in Sobolev spaces; we obtain sharp results with a random process somewhat like Brownian motion.
\end{abstract}

\section{INTRODUCTION}

We investigate dimension-increasing properties of mappings in the space $W^{1, p}\left(R^{n}\right), p>n \geq 1$, obtaining exact results as far as the Hausdorff dimension $(\operatorname{dim})$ and the packing dimension (Dim) are concerned. The upper bounds are consequences of Hölder's inequality; the lower bounds for $n>1$ depend on inequalities from probability theory like those governing Brownian motion.

All functions in $W^{1, p}\left(R^{n}\right), p>n \geq 1$, are assumed to be continuous. When $0<\alpha<n$ and $n<p<+\infty$, we define $\beta=(p \alpha)(p-n+\alpha)^{-1}$, so that $\alpha<\beta<n$.

Theorem 1. Let $E$ be a subset of $R^{n}$ of finite $\alpha$-dimensional measure, and let $f \in W^{1, p}\left(R^{n}\right)$. (The range of $f$ can have any dimension, even infinite.) Then $f(E)$ has $\beta$-measure 0 .

Theorem 2. Let $E$ be a closed subset of $R$, of positive $\alpha$-measure, $0<\alpha<1$. Then there is a real function $f$ on $R$, such that $f^{\prime}$ is in weak $L^{p}$, and $f(E)$ has positive $\beta$-measure.

Theorem 3. Let $E$ be a closed subset of $R^{n}$, of positive $\alpha$-measure, $0<\alpha<n$. Then $\operatorname{dim} f(E) \geq \beta$ for some $f \in W^{1, p}\left(R^{n}\right)$ mapping $R^{n}$ to $R^{n}$.

Similar bounds and existence theorems are obtained afterwards for the packing dimension [1], [2], [4], [5]. In this part the exposition is less formal.

\section{UPPER BOUNDS FOR $\operatorname{dim} f(E)$}

Constant use is made of the usual system $Q_{N k}(N=0, \pm 1, \pm 2, \ldots, k \geq 1)$ of dyadic $n$-cubes in $R^{n}$. These can be used in estimating Hausdorff measures in $R^{n}$ at the expense of a constant $c_{n}$. Let $Q$ be a cube of side $r$, and $f \in W^{1, p}\left(R^{n}\right)$. Then $f(Q)$ has diameter at most $c_{n, p} r^{1-n / p}\left(\int_{Q}\|\Delta f\|^{p} d m\right)^{1 / p}$. This is just an invariant form of a Sobolev inequality [3, p. 124]; it is valid for mappings into normed spaces.

Received by the editors January 30, 1998.

2000 Mathematics Subject Classification. Primary 28A12, 26B35; Secondary 60G50, 60G57, $26 \mathrm{~B} 15$.

Key words and phrases. Dimension, Sobolev spaces, random series, energy. 
To prove Theorem 1 we cover $E$ by cubes $Q_{j}$ of side $r_{j}$, so that $\sum r_{j}^{\alpha} \leq c(E)$ and $\sup \left(r_{j}\right)$ is as small as we please. Then

$$
\begin{gathered}
\operatorname{diam}\left(f\left(Q_{j}\right)\right) \leq c r_{j}^{1-n / p}\left(\int_{Q_{j}}\|\Delta f\|^{p}\right)^{1 / p}, \\
\operatorname{diam}\left(f\left(Q_{j}\right)\right)^{\beta} \leq c^{\beta} r_{j}^{(1-n / p) \beta}\left(\int_{Q_{j}}\|\Delta f\|^{p}\right)^{\beta / p} .
\end{gathered}
$$

We use the fact that the cubes $Q_{j}$ are essentially disjoint. We sum the left side over $j$, using Hölder's inequality with exponent $q=p \beta^{-1}$ for the factors on the right. The conjugate exponent is $q^{\prime}=p(p-\beta)^{-1}$; and finally $q^{\prime}\left(1-n p^{-1}\right) \beta=\alpha$. Since the union $\bigcup Q_{j}$ has measure $0(1) \sup \left(r_{j}\right)^{n-\alpha}$, we obtain $o(1)$ for the sum $\sum \operatorname{diam}\left(f\left(Q_{j}\right)\right)^{\beta}$.

\section{Proof of Theorem 2}

By Frostman's Theorem, $E$ carries a probability measure $\mu$ such that $\mu(I) \leq$ $c|I|^{\alpha}$ for all intervals $I$ and some constant $c$. We shall find a strictly increasing function $f$ such that $|f(I)| \geq \mu(I)^{1 / \beta}$ for all intervals $I$. Let $f^{*}(\mu)$ be the image of $\mu$ by the mapping $f: \mu^{*}(S) \equiv \mu\left(f^{-1}(S)\right)$ for open sets $S$. When $S$ is an interval, so is $f^{-1}(S)$ and it is evident that $M^{*}(I) \leq|I|^{\beta}$ for all intervals $I$, so that $f(E)$ has positive $\beta$-measure.

To find $f$ we require that $f^{\prime} \geq \mu(I)^{1 / \beta}|I|^{-1}$ on every open interval $I$, and prove that this can be done with some $f^{\prime}$ in weak $L^{p}$. We define $h(x)$ to be the supremum of $\mu(I)^{1 / \beta}|I|^{-1}$ over all intervals $I$ containing $x$. To prove that $h$ is in weak $L^{p}(R)$, we introduce $h(x, t)=\sup \mu(I)^{t}|I|^{-1}$, where $\alpha^{-1} \leq t \leq 1$. Then $h(x, 1)$ is the Hardy-Littlewood maximal function of $\mu$, and is in weak $L^{1}$. Again, $h\left(x, \alpha^{-1}\right)$ is bounded, so that $h(x)=h\left(x, \beta^{-1}\right)$ is in weak $L^{p}$, because $\beta^{-1}=p^{-1}+\alpha^{-1}\left(1-p^{-1}\right)$. This completes Theorem 2; to obtain $f^{\prime} \in L^{p}$ and $\operatorname{dim} f(E) \geq \beta$, we decrease $h$ slightly to $h^{\sim}(x)=\sup \mu(I)^{1 / \beta}|I|^{-1} \log ^{-1}\left(e+|I|^{-1}\right)$.

\section{3}

For Theorem 3 we use the system $Q_{N k}$ of dyadic squares of side $2^{-N}$, where $N=0, \pm 1, \pm 2, \ldots$ and $k=1,2,3, \ldots$. These define a sequence of bump functions $\psi_{N k}$, equal to 1 on $Q_{N k}$, and vanishing outside the cube $Q_{N k}^{\sim}$ obtained by expanding $Q_{N k}$ on its center by a factor $5 / 4$; moreover $0 \leq \psi_{N k} \leq 1$ and $\left\|\nabla \psi_{N k}\right\| \leq c_{n} 2^{N}$. For a sum $u=\sum_{k} c_{k} \psi_{N k}$ we find $\|\nabla u\|_{p}^{p} \approx 2^{N p} 2^{-n N} \sum\left|c_{k}\right|^{p}$. Let $\mu$ be a probability measure in $R^{n}$, satisfying the Hölder condition in exponent $\alpha$ and $c_{k}=\mu\left(Q_{N k}\right)^{1 / \beta}$. We claim that $\|\nabla u\|_{p}^{p}$ is bounded by a number independent of $k$; here $\alpha, \beta, n, p$ are the numbers defined before. In fact $\sum_{k} \mu\left(Q_{N k}\right) \leq C_{1}$ and $\sup \mu\left(Q_{N k}\right) \leq C_{2} 2^{-N \alpha}$. Thus $\sum_{k} c_{k}^{p} \leq C_{3} 2^{-N \gamma}$ where $\gamma=\alpha\left(p \beta^{-1}-1\right)$. To complete our claim we observe that $p-n=\gamma$.

In fact we use a more involved variant of the function $u$. First we replace $\mu\left(Q_{N k}\right)$ by $\mu\left(Q_{N k}^{* *}\right)$, where $Q_{N k}^{* *}$ is obtained from $Q_{N k}$ by expansion in a ratio of 8 . Moreover we introduce a sequence of random multipliers $\xi_{N k}$, independent random variables uniformly distributed on the unit ball in $R^{n}$. We call the variant so obtained $v_{N}$ and observe that the estimates on $\nabla v_{N}$ are almost the same as the ones above.

Suppose now that $E$ is contained in the unit cube of $R^{n}$, and that $\mu$ is concentrated in $E$. We define $v=\sum_{0}^{\infty}(N+1)^{-2} v_{N}$, and prove that the support of $v^{*}(\mu)$ 
has Hausdorff dimension at least $\beta$ a.s., as follows. Let $k(t)=t^{-\beta} \log ^{-2 n-2}\left(e+t^{-1}\right)$ for $t>0$. Then the energy of $v^{*}(\mu)$ with respect to the kernel $k$ is

$$
I=\iint k(v(x)-v(y)) \mu(d x) \mu(d y) .
$$

If $I<+\infty$, then $v^{*}(\mu)$ vanishes on sets of dimension $<\beta$; we prove that $\mathcal{E}(I)<+\infty$, whence our assertion on the support of $v^{*}(\mu)$ follows.

In fact we prove that $\int \mathcal{E}(k(v(x)-v(y))) \mu(d y) \leq c<+\infty$ for all $x$ in the unit cube, whence $\mathcal{E}(I)<+\infty$ by Fubini's Theorem. For each $x$ and $y, v(x)-$ $v(y)=\sum \sum a_{N k}(x, y) \xi_{N k}$; the variables $\xi_{N k}$ are those described above. Let $\rho(x, y)=\sup \left|a_{N k}(x, y)\right|$ so that $0 \leq \rho \leq 2$. We easily see that the expectation of $k(v(x)-v(y))$ is $\leq c k(\rho)$, since $0<\beta<n$. Thus we seek a uniform upper bound for $\int k(\rho(x, y)) \mu(d y)$.

Let $\nu(x, y)$ be the largest integer $N \geq 0$ such that $x$ and $y$ belong to a single square $Q_{N k}$ or to touching squares of side $2^{-N}$. (When $x \neq y$, then $\nu(x, y)<$ $+\infty$.) Then there will be a bump function $\psi_{N+1}$, of the next generation, such that $\psi_{N+1}(x)=1, \psi_{N+1}(y)=0$. More precisely, $x \in Q_{N+1, \ell}, y \notin Q_{N+1, \ell}^{\sim}$; however $y \in Q_{N+1, \ell}^{* *}$. Thus, if we partition the domain of integration into the sets defined by $\nu(x, y)=0, \nu(x, y)=1, \ldots, \nu(x, y)=N, \ldots$, then the $\mu$-measure of the $N^{t h}$ set is $<\mu\left(Q_{N+1, \ell}^{* *}\right) \equiv \mu_{N+1}$ but for each $y$ in the set $\rho(x, y) \geq(N+1)^{-2} \mu_{N+1}^{1 / \beta}$. Moreover $\mu_{N+1}<c 2^{-2 N \alpha}$. Observing the logarithm in $k(t)$ we see that the integral of $k(\rho(x, y))$ on the $N^{t h}$ set is $0\left(N^{-2}\right)$, since the logarithm adds a factor $c N^{-2 n-2}$, which balances the factor $N^{2 \beta}$ arising from the formula for $v$. We observe also that $\nu(x, y) \geq 0$ because $E$ is in the unit cube, and when $\nu(x, y)=0$, then $\rho(x, y)=1$. Thus we have verified the estimate on $\int \mathcal{E}(k(v(x)-v(y))) \mu(d y)$ and Theorem 3 is completely proved.

\section{PACKing Dimension}

This concept is derived from the more precise notion of packing measure in much the same way as Hausdorff dimension is derived from Hausdorff measure; thus the following definition is part of a larger picture, [1], [2, pp. 82-86], [4]. Let $S$ be a set in a metric space and $r>0$; then $\nu(S, r)$ is the smallest number of sets of diameter at most $r$, sufficient to cover $S$. The packing exponent $\delta(S)=$ $\lim \sup \nu(S, r) /-\log r, r \rightarrow 0+$. (This notion is standard, but the name is not.) A variant concept is the number $\nu^{*}(S, r)$, the largest number of elements of $S$ separated by more than $r$. Then $\nu^{*}(S, R) \leq \nu(S, r) \leq \nu^{*}\left(S, \frac{1}{2} r-\right)$, so that $\nu^{*}$ can be used to define $\delta(S)$. We say that $S$ has packing dimension $\leq \alpha, \operatorname{Dim} S \leq \alpha$, if for each $\epsilon>0, S=\bigcup_{1}^{\infty} S(m, \epsilon)$ where $\delta(S(m, \epsilon))<\alpha+\epsilon$.

Theorem 4. Let $f \in W^{1, p}\left(R^{n}\right), E \subseteq R^{n}$, and $\operatorname{Dim} E \leq \alpha$. Then $\operatorname{Dim} f(E) \leq \beta$.

Proof. In calculating the exponent of packing of a set in $E^{n}$, we can use dyadic squares of the same side $2^{-N}$. Let $\gamma=\alpha \beta^{-1}$ and $r=2^{-N \gamma}$. A cube $Q$ of side $2^{-s}, s \geq N$, is called major if diam $f(Q) \geq r$, minor in the opposite case, and critical if it is major, but each of its descendants is minor. Thus a critical cube is the union of $2^{n}$ minor cubes. Therefore critical cubes play almost the same role as minor cubes, except that they can be counted by counting the number of major cubes. Since $f$ is uniformly continuous in $R^{n}$, major cubes can be subdivided successively until critical cubes are encountered. We shall first count all major 
cubes; in spite of its apparent inefficiency, this gives the correct estimate of packing dimension.

The number $m_{p}$ of major cubes of side $2^{-s}$ is estimated by Sobolev's inequality: $m_{p} \leq c r^{-p} 2^{-s(p-n)}$. Since $p>n$, the sum is at most $c^{\prime} r^{-p} 2^{-N(p-n)}=c^{\prime} 2^{\alpha N}$ since $\alpha p \beta^{-1}-(p-n)=\alpha$.

Suppose that a set $B$ has packing exponent $<\eta$, so that for large $N B$ is covered by at most $2^{\eta N}$ cubes of side $2^{-N}$. Then $B$ is covered by the minor cubes of side $2^{-N}$, augmented by all of the critical cubes of side $2^{-N}$ or smaller. The number of cubes in this covering is $0\left(2^{\eta N}+2^{\alpha N}\right)$, whence $f(B)$ has packing exponent at most $\max \left(\beta, \eta \alpha^{-1} \beta\right)$. For each $\eta>\alpha, E$ is contained in a sequence of sets of packing exponent $<\eta$, whence $\operatorname{Dim} f(E) \leq \max \left(\beta, \eta \alpha^{-1} \beta\right)$, or $\operatorname{Dim} f(E) \leq \beta$.

Theorem 5. Let $E$ be a closed set in $R^{n}$ and $\operatorname{Dim} E>\alpha$. Then there is a mapping $h$ of class $W^{1, p}\left(R^{n}\right)$ into $R^{n}$, such that $\operatorname{Dimh}(E) \geq \beta$.

With more attention to inequalities, Theorem 4 can be proved with a weaker hypothesis, $\operatorname{Dim} E \geq \alpha$. Using the separability of $R^{n}$ we can find a closed subset $E_{0} \neq \emptyset$, such that every relatively open subset $W_{0} \neq \emptyset$ of $E_{0}$ has $\operatorname{Dim} W_{0}>\alpha$. Henceforth we replace $E$ by $E_{0}$ in the argument. In place of energy integrals, appearing in Theorem 3, we employ the following consequence of Cauchy's inequality. A set $B$ has $m$ distinct elements $x_{1}, \ldots, x_{m}$ and is partitioned into $k$ subsets $T_{1}, \ldots, T_{k}$. Then the number $N$ of pairs $\left(x_{i}, x_{j}\right)$ such that $x_{i}$ and $x_{j}$ belong to the same set $T_{\nu}, 1 \leq \nu \leq k$, is at least $m^{2} / k$. Counting the number $N_{0}$ of pairs in which $x_{i} \neq x_{j}$, we obtain $N_{0} \geq m^{2} / k-m$.

Next we describe a basic step in Theorem 5 , with some heuristics. The set $B$ is located in the unit cube of $R^{n}$, and its elements are separated by $r, 0<r<e^{-2}$, while the size $m$ of $B$ is $\left[r^{-\alpha}\right]$. We seek a random function $f$ so that nearly all of the images $f\left(x_{i}\right), 1 \leq i \leq m$, are separated by $r^{\gamma}, \gamma=\alpha \beta^{-1}$. (This would reverse the inequalities obtained in Theorem 4.) A cube $Q$ containing $\ell \geq 2$ of the points $x_{i}$ should be mapped on a set of diameter $\geq c r^{\gamma} \ell^{1 / n}$. This suggests the following choice of coefficients in the random function $f$; only numbers $N$ such that $1 \geq 2^{-N} \geq r / 8 n$ are used in the summation. Let $\lambda$ be the counting measure of the set $B$, and the coefficient of $c_{N k}$ of $\psi_{N k}$ be $r^{\gamma} \lambda\left(Q^{* *}\right)^{1 / n}$. First we have to verify the $W^{1 / p}$-type inequality for the indicated values of $N$, i.e. $\sum_{k} c_{N k}^{p} \leq c 2^{N(n-p)}$ or $\sum_{k} \lambda\left(Q_{N k}^{* *}\right)^{p / n} \leq c r^{-\gamma p} 2^{N(n-p)}$. Now the total mass of $\lambda$ is $<r^{-\alpha}$ and $\lambda\left(Q_{N k}^{* *}\right) \leq$ $c\left(2^{-N} r^{-1}\right)^{n}$ since $2^{-N} \geq r / 8 n$. We obtain $\sum_{k} \lambda\left(Q_{N k}^{* *}\right)^{p / n} \leq c r^{-\alpha}\left(2^{-N} r^{-1}\right)^{(p-n)}$, and this suffices because $-\alpha+n-p=-\gamma p$. (We don't use the factors $(N+1)^{-2}$ in the sum, so there is a small correction later.)

By the method of estimation used in Theorem 3, we find that the expected number of pairs $\left(x_{i}, x_{j}\right)$, such that $i \neq j$ and $\left|f\left(x_{i}\right)-f\left(x_{j}\right)\right|<r^{\gamma}$, is $0(m \log 1 / r)$. Here we used the requirement that $\left|x_{i}-x_{j}\right| \geq r$ when $i \neq j$. The $W^{1, p}$-norm of the $\operatorname{sum} \sum_{N} \sum_{k} c_{N k} \xi_{N k} \psi_{N k}$ is $0(\log 1 / r)$. Outside a set of measure $c \log ^{-2}(1 / r)$, the number of pairs referred to above is $\leq m \log ^{3} 1 / r$. Thus

$$
\nu\left(f(B), r^{\gamma}\right) \geq \frac{1}{2} r^{-\alpha} \log ^{-3}(1 / r) .
$$

The same estimate for the exceptional set is valid for a sum $f+g$, provided $f$ and $g$ are independent. 
Returning to the set $E_{0}$, which we can assume is contained in the unit square, we find a sequence of sets $B_{j}$ of $E_{0}$, and numbers $r_{j}$ such that $r_{j}<\exp -j^{-2}$, $B_{j}$ has size $\left[r_{j}^{-\alpha}\right]$, and its elements are separated by $r_{j}$. Moreover, every open set $W \neq \emptyset$ of $E_{0}$ contains infinitely many of the sets $B_{j}$. For each $j$ we define the random function $f_{j}$ as above and then define $h=\sum_{1}^{\infty} j^{-2} \log ^{-1}\left(1 / r_{j}\right) f_{j}$, a series converging in $W^{1, p}\left(R^{n}\right)$, with independent terms. Except for a set of measure $0\left(j^{-4}\right)$, we have $\nu\left(h\left(B_{j}\right), r_{j}^{\gamma} j^{-2} \log ^{-1}\left(1 / r_{j}\right)\right)>c r_{j}^{-\alpha} \log ^{-3}\left(1 / r_{j}\right)$.

We can now prove that $\operatorname{Dim} h\left(E_{0}\right) \geq \beta$ almost surely, using an observation from [1]. In the opposite case $h\left(E_{0}\right)$ is a countable union of sets $A_{j}$, of packing exponent $<\beta$. Since $A_{j}$ and its closure have the same exponents, we conclude with the aid of the Baire Category Theorem that some open set $V \neq \emptyset$ in $h(E)$ has packing exponent $<\beta$. But then $h^{-1}(V)$ is relatively open in $E_{0}$, so that $V$ contains infinitely many of the sets $h\left(B_{j}\right)$. This contradicts the almost-sure estimates on the packing numbers of the sets $h\left(B_{j}\right)$, proving Theorem 5 .

\section{REFERENCES}

1. Haase, H., Non- $\sigma$-finite sets for packing measure, Mathematika 33 (1986), 129-136. MR 88a:28003

2. Mattila, P., Geometry of Sets and Measures in Euclidean Spaces, Cambridge studies $\underline{44}$, Cambridge University Press (1995). MR 96h:28006

3. Stein, E. Singular integrals and differentiability properties of functions, Princeton University Press (1970). MR 44:7280

4. Taylor, S.J. and Tricot, C., Packing measure and its evaluation for a Brownian path, Trans. Amer. Math. Soc. 288 (1985), 679-699. MR 87a:28002

5. Tricot, C., Two definitions of fractional dimension, Math. Proc. Camb. Phil. Soc. 91 (1982), 57-74. MR 84d:28013

Department of Mathematics, University of Illinois, 1409 West Green Street, UrBANA, ILLINOIS 61801

E-mail address: rpkaufma@math.uiuc.edu 